\title{
Convex Interpolation by Splines of Arbitrary Degree
}

\author{
By Holger Mettke
}

\begin{abstract}
An algorithm is described for computing an interpolation spline of arbitrary but fixed degree which preserves the convexity of the given data set. Necessary and sufficient conditions for the solvability of the problem, some special cases and error estimations are given.
\end{abstract}

1. Introduction. In some problems arising from science or engineering the solution of an interpolation problem with constraints is required. For example, if a convex data set is given then the interpolant should also be convex. Using standard techniques like polynomial or cubic spline interpolation the resulting interpolant is not convex in general (see [5]).

In recent years convex interpolation by splines has been investigated in several publications (see [3]-[17]). Various possibilities were proposed to assure the convexity of an interpolation spline. They reach from additional conditions on the data set to additional knots of the interpolation spline.

In this paper we consider convex interpolation by splines of arbitrary degree $k \geqslant 3$ with smoothness $q$, where $1 \leqslant q \leqslant[(k-1) / 2]$. As is known, the solvability of such an interpolation problem is equivalent to that of a special system of linear inequalities. Necessary and sufficient conditions are derived such that this system has a solution, and an algorithm is described to find all solutions of the system of inequalities. Furthermore, a sufficient condition is given which always assures the solvability of the system and which is easy to test. In this case, a solution can be found even with a reduced algorithm. The sufficient condition just mentioned is interpreted in different ways. Specifically, for a strictly convex data set it is possible to give a lower bound for the degree $k$ such that the interpolation problem is solvable. For the developed method no additional spline knots are needed. In the last section, the important question of error estimation is investigated. Under certain assumptions, error bounds are derived for continuous and continuously differentiable underlying functions. The assumptions are related to the solvability of the convex interpolation problem, and in one case to the ratio of contiguous step sizes of the grid. In the differentiable case, the order of approximation is better than in the continuous one.

2. Definitions and Notation. Let $\pi$ be an arbitrary but fixed partition of the interval $[a, b]$, i.e.,

$$
\pi: a=x_{0}<x_{1}<\cdots<x_{n-1}<x_{n}=b \quad(n>0) .
$$

Received February 3, 1984; revised June 6, 1985.

1980 Mathematics Subject Classification. Primary 65D05, 41A15.

C1986 American Mathematical Society $0025-5718 / 86 \$ 1.00+\$ .25$ per page 
The maximal mesh length is abbreviated as

$$
h=\max _{1 \leqslant i \leqslant n} h_{i} \text { where } h_{i}=x_{i}-x_{i-1} \text {. }
$$

Further, for given integers $k, q$ with $0 \leqslant q \leqslant k-1$ we denote by $S(k, q, \pi)$ the space of all polynomial splines of degree $k$ associated with $\pi$ with deficiency at most $(k-q)$ at the knots. That is, $s \in S(k, q, \pi)$ if and only if $s \in C^{q}[a, b]$ and in each subinterval $\left[x_{i-1}, x_{i}\right] s$ coincides with a polynomial of degree $k$.

Let $f_{i}(i=0,1, \ldots, n)$ be given real numbers. Then the data set $\left\{\left(x_{i}, f_{i}\right)\right.$, $i=0,1, \ldots, n\}$ is said to be convex if and only if, with the abbreviation $\tau_{i}=$ $\left(f_{i}-f_{i-1}\right) / h_{i}$, the inequalities

$$
\tau_{1} \leqslant \tau_{2} \leqslant \cdots \leqslant \tau_{n-1} \leqslant \tau_{n}
$$

are valid (see [14], [15]). The data set is called strictly convex if and only if the inequalities $\tau_{1}<\tau_{2}<\cdots<\tau_{n-1}<\tau_{n}$ hold. Our aim is to investigate the following interpolation problem (I).

(I) For a given convex data set $\left\{\left(x_{i}, f_{i}\right), i=0,1, \ldots, n\right\}$ find a spline $s \in S(k, q, \pi)$ satisfying the conditions

$$
s\left(x_{i}\right)=f_{i} \quad(i=0,1, \ldots, n)
$$

and

$$
s \text { is convex on }[a, b] .
$$

In this paper we restrict our attention to the case when $k \geqslant 3$ and $1 \leqslant q \leqslant[(k-1) / 2]$ where, as usual, $[r]$ denotes the greatest integer not greater than $r$.

In order to construct solutions of (I), some special fundamental polynomials are used. They are defined in the following manner (see [12] and for special cases [1], [13], [18]): Let $u_{i}(i=1,2, \ldots, n)$ be given integers such that $1<u_{i}<k$ and $\min _{1 \leqslant j \leqslant n-1} r_{j}=q$, where $r_{j}=\min \left\{u_{j}-1, k-u_{j+1}\right\}$. Then the functions

$$
\begin{aligned}
& \varphi_{1, i}(t)=a_{k, u_{i}} \cdot \int_{0}^{t} \xi^{k-u_{i}}(1-\xi)^{u_{i}-1} d \xi, \\
& \varphi_{0, i}(t)=1-\varphi_{1, i}(t), \\
& \varphi_{2, i}(t)=t \cdot\left[1-a_{k-1, u_{i}} \cdot \int_{0}^{t} \xi^{k-u_{i}-1}(1-\xi)^{u_{i}-1} d \xi\right], \\
& \varphi_{3, i}(t)=a_{k-1, u_{i}-1}(1-t) \cdot \int_{0}^{t} \xi^{k-u_{i}}(1-\xi)^{u_{i}-2} d \xi
\end{aligned}
$$

$(i=1,2, \ldots, n)$, defined on $[0,1]$ are the fundamental polynomials. Here, the abbreviation $a_{\mu, \nu}$ means

$$
a_{\mu, \nu}=\left(\begin{array}{c}
\mu \\
\nu
\end{array}\right)=\left[\int_{0}^{1} \xi^{\mu-\nu}(1-\xi)^{\nu-1} d \xi\right]^{-1}
$$

For the reader's convenience we recall some properties of these functions:

(i) $\varphi_{0, i}, \ldots, \varphi_{3, i}$ are polynomials of degree $k$.

(ii) $\varphi_{j, i}:[0,1] \rightarrow[0,1], j=0,1,2,3$.

(iii) $\varphi_{j, i}$ is determined by the following fundamental properties:

$$
\begin{array}{ll}
\varphi_{j, i}^{(\nu)}(0)=\delta_{j, 0} \delta_{\nu, 0}, & \varphi_{j+2, i}^{(\nu)}(0)=\delta_{j, 0} \delta_{\nu, 1} \quad\left(j=0,1 ; \nu=0, \ldots, k-u_{i}\right), \\
\varphi_{j, i}^{(\nu)}(1)=\delta_{j, 1} \delta_{\nu, 0}, & \varphi_{j+2, i}^{(\nu)}(1)=(-1)^{j} \delta_{j, 1} \delta_{\nu, 1} \quad\left(j=0,1 ; \nu=0, \ldots, u_{i}-1\right) .
\end{array}
$$


Thus, the above polynomials are special Hermite interpolation polynomials. We define

$$
p_{i}(x)=f_{i-1} \varphi_{0, i}(t)+f_{i} \varphi_{1, i}(t)+h_{i}\left[m_{i-1} \varphi_{2, i}(t)-m_{i} \varphi_{3, i}(t)\right]
$$

$\left(x \in\left[x_{i-1}, x_{i}\right]\right)$, with the abbreviation

$$
t=\frac{x-x_{i-1}}{h_{i}}
$$

which is used from here on throughout the paper. The quantities $m_{i}=p_{i}^{\prime}\left(x_{i}\right)=$ $p_{i+1}^{\prime}\left(x_{i}\right)$ are free parameters. Then the function $s:[a, b] \rightarrow R$, given by

$$
s(x)=p_{i}(x) \quad\left(x \in\left[x_{i-1}, x_{i}\right]\right),
$$

is a spline from $S(k, q, \pi)$. Moreover, for every choice of parameters $m_{i}$ the spline $s$ solves the interpolation problem (2.1). We note that in particular $s \in C^{r_{i}}$ in a neighborhood of $x_{i}$. Hence the deficiency of $s$ at the knot $x_{i}$ can be controlled with the help of $u_{i}, u_{i+1}$.

3. Convex Interpolation. In general, for a convex data set an interpolating spline of the form (2.4), (2.5) is not convex. There arises the question if there exist parameters $m_{i}$ such that the corresponding interpolating spline $s$ is also a solution of (2.2). A partial answer to this question is given by the following theorem (see [12]).

Theorem 3.1. Let $\left\{\left(x_{i}, f_{i}\right), i=0, \ldots, n\right\}$ be a convex data set. Then an interpolating spline $s$ of the form (2.4), (2.5) is convex on the interval $[a, b]$ if and only if the parameters $m_{0}, m_{1}, \ldots, m_{n}$ solve the linear system of inequalities

$$
\begin{array}{r}
\left(k-u_{i}+1\right) m_{i-1}+\left(u_{i}-1\right) m_{i} \leqslant k \tau_{i} \\
\left(k-u_{i}\right) m_{i-1}+u_{i} \cdot m_{i} \geqslant k \tau_{i}
\end{array} \quad(i=1,2, \ldots, n) .
$$

If we can find a solution of (3.1) then, by (2.4), (2.5), a solution of (I) is given. On the other hand, there exist convex (even strictly convex) data sets for which the system (3.1) is unsolvable. This is a consequence of the following theorem, given by Passow and Roulier [15]:

THEOREM 3.2. For any integer $k \geqslant 1$ there exists a set of five data points $\left\{\left(x_{i}, f_{i}\right)\right.$, $i=0, \ldots, 4\}$ with $\tau_{1}<\tau_{2}<\tau_{3}<\tau_{4}$, for which no spline $s \in S(k, 1, \tilde{\pi}), \tilde{\pi}: x_{0}<x_{1}$ $<x_{2}<x_{3}<x_{4}$ satisfies $s\left(x_{i}\right)=f_{i}(i=0, \ldots, 4)$ with s convex on $\left[x_{0}, x_{4}\right]$.

4. An Algorithm. In this section we present an algorithm for solving (3.1). Moreover, necessary and sufficient conditions for the solvability of this system are derived. In [6] the author described an algorithm for solving (3.1) in the case $k=3$. The extension of the method to the general system (3.1) was realized in an unpublished paper. Starting from this, Schmidt and Hess [17] developed a more effective variant. The algorithm given here is the symmetric variant of it. For simplification, in view of (3.1), we use the following abbreviations:

$$
\alpha_{i}=\left(k-u_{i}+1\right) / k, \quad \beta_{i}=\left(u_{i}-1\right) / k, \quad \gamma_{i}=\left(k-u_{i}\right) / k, \quad \delta_{i}=u_{i} / k .
$$




\section{AlgorithM}

Step 1 (Backward-step):

Put $A_{n}=\tau_{n}$ and $B_{n}=\left(\tau_{n}-\alpha_{n} \tau_{n-1}\right) / \beta_{n}$

For $i=n, n-1, \ldots, 1$ do

If $\tau_{i}>B_{i}$ then stop else

put $A_{i-1}=\left(\tau_{i}-\delta_{i} B_{i}\right) / \gamma_{i}$ and $B_{i-1}=\min \left\{\tau_{i},\left(\tau_{i}-\beta_{i} A_{i}\right) / \alpha_{i}\right\}$

Step 2 (Forward-step):

Choose $m_{0} \in\left[\underline{m}_{0}, \bar{m}_{0}\right]$ with $\underline{m}_{0}=A_{0}$ and $\bar{m}_{0}=B_{0}$.

For $i=1,2, \ldots, n$ do

Choose $m_{i} \in\left[\underline{m}_{i}, \bar{m}_{i}\right]$ with

$$
\begin{aligned}
\underline{m}_{i} & =\max \left\{A_{i},\left(\tau_{i}-\gamma_{i} m_{i-1}\right) / \delta_{i}\right\} \text { and } \\
\bar{m}_{i} & =\min \left\{B_{i},\left(\tau_{i}-\alpha_{i} m_{i-1}\right) / \beta_{i}\right\} .
\end{aligned}
$$

Before we make some remarks on the Algorithm let us formulate the following result, which can be proved by considerations analogous to those in [17].

THEOREM 4.1. Let the data set $\left\{\left(x_{i}, f_{i}\right), i=0,1, \ldots, n\right\}$ be convex. Then the following statements are equivalent:

(i) The system of inequalities (3.1) is solvable.

(ii) The Algorithm is well-defined and yields a solution of (3.1).

(iii) The inequalities $\tau_{i} \leqslant B_{i}$ are valid for $i=1,2, \ldots, n$.

Remarks. 1. The above Algorithm makes strong use of the special structure of the system (3.1).

2. The Algorithm is of a global nature in the sense that a change of data points or an extension of the data set has influence on all intervals of admissible parameters in general.

3. The fact that we have a whole interval $\left[\underline{m}_{i}, \bar{m}_{i}\right]$ of admissible parameters $m_{i}$ can be used to look for "visually pleasant" convex spline interpolants (see [2]).

4. It is easy to show that with the help of the Algorithm all solutions of (3.1) can be found.

5. The statement (iii) in Theorem 4.1 can be replaced by '(iii') The inequalities $\tau_{i} \leqslant B_{i}$ are valid for $i=1,2, \ldots, n-3$." This is possible because the remaining inequalities always hold.

5. Special Cases. The first special case is embodied in the following theorem.

THEOREM 5.1. Let the convex data set $\left\{\left(x_{i}, f_{i}\right), i=0,1, \ldots, n\right\}$ be given such that

$$
\frac{u_{j+1}}{k-u_{j+1}} \cdot\left(\tau_{j+2}-\tau_{j+1}\right)-\left(\tau_{j+1}-\tau_{j}\right) \geqslant 0, \quad j=1,2, \ldots, n-2 .
$$

Then there exists a solution of (3.1) and, consequently, the interpolation problem (I) possesses a solution.

Proof. Because of Theorem 4.1 it is sufficient to verify that $\tau_{i} \leqslant B_{i}(i=1,2, \ldots$, $n-3)$. This follows from the next lemma, which completes the proof. Q.E.D.

LEMMA 5.2. With the assumption of Theorem 5.1 we have

$$
B_{i-1}=\tau_{i}, \quad i=1,2, \ldots, n .
$$


Proof. For $i=n, n-1$ the relation (5.2) is valid. Let us suppose that (5.2) holds for $i=n, n-1, \ldots, j+1$. Then $B_{j} \geqslant \tau_{j}$ and $A_{j-1}, B_{j-1}$ are well-defined. Because of

$$
A_{j}=\frac{\tau_{j+1}-\delta_{j+1} B_{j+1}}{\gamma_{j+1}}=\frac{\tau_{j+1}-\delta_{j+1} \tau_{j+2}}{\gamma_{j+1}},
$$

we obtain, with (5.1), that

$$
\begin{aligned}
\frac{\tau_{j}-\beta_{j} A_{j}}{\alpha_{j}}-\tau_{j} & =\frac{\beta_{j}}{\alpha_{j}}\left(\tau_{j}-A_{j}\right) \\
& =\frac{\beta_{j}}{\alpha_{j}}\left\{\frac{\delta_{j+1}}{\gamma_{j+1}}\left(\tau_{j+2}-\tau_{j+1}\right)-\left(\tau_{j+1}-\tau_{j}\right)\right\} \geqslant 0 .
\end{aligned}
$$

Thus $B_{j-1}=\tau_{j}$. Q.E.D.

In the case of (5.1) the backward-step of the Algorithm can be omitted and we get a

\section{Reduced Algorithm.}

For $i=1,2, \ldots, n$ choose $m_{i} \in\left[\underline{m}_{i}, \bar{m}_{i}\right]$ with

$$
\begin{aligned}
& \underline{m}_{0}=\frac{\tau_{1}-\delta_{1} \tau_{2}}{\gamma_{1}}, \quad \bar{m}_{0}=\tau_{1} \\
& \underline{m}_{j}=\max \left\{\frac{\tau_{j+1}-\delta_{j+1} \tau_{j+2}}{\gamma_{j+1}}, \frac{\tau_{j}-\gamma_{j} m_{j-1}}{\delta_{j}}\right\} \\
& \bar{m}_{j}=\min \left\{\tau_{j+1}, \frac{\tau_{j}-\alpha_{j} m_{j-1}}{\beta_{j}}\right\} \\
& \underline{m}_{n-1}=\frac{\tau_{n-1}-\gamma_{n-1} m_{n-1}}{\delta_{n-1}} \quad(j=1,2, \ldots, n-2) \\
& \bar{m}_{n-1}=\min \left\{\tau_{n}, \frac{\tau_{n-1}-\alpha_{n-1} m_{n-2}}{\beta_{n-1}}\right\} \\
& \underline{m}_{n}=\frac{\tau_{n}-\gamma_{n} m_{n-1}, \quad \bar{m}_{n}=\frac{\tau_{n}-\alpha_{n} m_{n-1}}{\delta_{n}} .}{\beta_{n}} .
\end{aligned}
$$

Remark. If we put $k=3$ and, hence, $u_{i}=2(i=1,2, \ldots, n)$ the above Reduced Algorithm is equivalent to Algorithm 2 presented in [6]. From Theorem 5.1 we can derive some interesting conclusions. We first assume that the degree $k$ is fixed and the smoothness $q, q \geqslant 1$, is not of importance, i.e., the parameters $u_{i}$ can be chosen arbitrarily between 1 and $k$. Then we get

COROllaRy 5.3. Let the given data set be strictly convex. If parameters $u_{i}$, $(i=2,3, \ldots, n-1)$, can be found with

$$
\frac{k\left(\tau_{j+1}-\tau_{j}\right)}{\tau_{j+2}-\tau_{j}} \leqslant u_{j+1}<k \quad(j=1,2, \ldots, n-2),
$$

then the interpolation problem (I) possesses a solution.

This fact is clear because the left-hand inequality of (5.3) is equivalent to (5.1). The remaining quantities, namely $u_{1}$ and $u_{n}$, have no influence on the result of Corollary 5.3. Therefore, they can be chosen arbitrarily with $1<u_{1}, u_{n}<k$. 
If for fixed $k$ the problem (I) has no solution then there arises the question: Does there exist a solution of (I) for splines of degree larger than $k$ ? An answer to this question was given implicitly in [5]. We obtain a positive answer with the help of (5.3) in the form of a lower bound for $k$. Namely, if for given $q \in\{1,2, \ldots$, $[(k-1) / 2]\}$

$$
\frac{k\left(\tau_{j+1}-\tau_{j}\right)}{\tau_{j+2}-\tau_{j}} \leqslant k-q \quad(j=1,2, \ldots, n-2),
$$

then we could take $u_{j+1}=k-q(j=1,2, \ldots, n-2)$ as admissible values and, hence, a $q$-times continuously differentiable solution of $(\mathrm{I})$ is guaranteed. From (5.4) we obtain (see also [17]):

COROllary 5.4. Let the given data set be strictly convex. If the degree $k$ of used splines is chosen such that

$$
k \geqslant q \cdot \max _{1 \leqslant j \leqslant n-2} \frac{\tau_{j+2}-\tau_{j}}{\tau_{j+2}-\tau_{j+1}},
$$

then there exists a solution of (I) in $S(k, q, \pi)$.

Obviously, under the assumptions of Corollary 5.3 resp. 5.4, corresponding solutions can be found with the help of the Reduced Algorithm. Corollary 5.4 is an alternative to the negative result of Theorem 3.2 of Passow and Roulier [15], given there for fixed $k$. Finally, we point out the fact that under assumption (5.1) a solution of (3.1) can be obtained by solving a first-order linear difference equation.

COROllary 5.5. For a given convex data set let (5.1) be valid. Then the solution of

$$
\gamma_{i} m_{i-1}+\delta_{i} m_{i}=\tau_{i} \quad(i=1,2, \ldots, n)
$$

with $m_{0}=\left(\tau_{1}-\delta_{1} \tau_{2}\right) / \gamma_{2}$ is also a solution of (3.1).

Proof. In view of (3.1) we have only to verify that the solution of (5.6) fulfills the inequalities

$$
\alpha_{i} m_{i-1}+\beta_{i} m_{i} \leqslant \tau_{i} \quad(i=1,2, \ldots, n) .
$$

From (5.6) we obtain after easy calculations

$$
\alpha_{i} m_{i-1}+\beta_{i} m_{i}=\tau_{i}+\frac{1}{k \gamma_{i}}\left(\tau_{i}-m_{i}\right)
$$

Thus we have to show that $\tau_{i}-m_{i} \leqslant 0(i=1,2, \ldots, n)$. We prove this by induction. For $i=1,2$ the relation is obviously fulfilled. For $j \geqslant 3$ we get

$$
\begin{aligned}
\tau_{j}-m_{j} & =\tau_{j}-\frac{\tau_{j}-\gamma_{j} m_{j-1}}{\delta_{j} \cdot}=\frac{\gamma_{j}}{\delta_{j} \delta_{j-1}}\left\{\tau_{j-1}-\delta_{j-1} \tau_{j}-\gamma_{j-2} m_{j-2}\right\} \\
& \leqslant \frac{\gamma_{j}}{\delta_{j} \delta_{j-1}}\left\{\tau_{j-1}-\delta_{j-1} \tau_{j}-\gamma_{j-1} \tau_{j-2}\right\} \leqslant 0,
\end{aligned}
$$

because of (5.1). Q.E.D.

The second special case deals with monotone increasing and convex spline interpolation. Given a monotone increasing and convex data set, i.e., $0 \leqslant \tau_{1} \leqslant \tau_{2} \leqslant$ $\cdots \leqslant \tau_{n}$, then we obtain with the Algorithm nonnegative solutions of (3.1) if the conditions (iii) of Theorem 4.1 and $B_{0} \geqslant 0$ hold and if in Step 2 of the Algorithm, 
$\underline{m}_{0}$ is substituted by

$$
\underline{m}_{0}=\max \left\{A_{0}, 0\right\} .
$$

If even (5.1) is valid, then the Reduced Algorithm always produces a nonnegative solution by substituting $\underline{m}_{0}$ by

$$
\underline{m}_{0}=\max \left\{\frac{\tau_{1}-\delta_{1} \tau_{2}}{\gamma_{1}}, 0\right\}
$$

Thus we obtain a monotone increasing and convex solution of (I).

6. Error Estimation. Let $f \in C[a, b]$ be a given function and let $f_{i}=f\left(x_{i}\right)$ $(i=0,1, \ldots, n)$. We denote by $\|f\|$ the maximum norm and by $\omega(f, \delta)$ the modulus of continuity of $f$ on the interval $[a, b]$. Further, let

$$
\tilde{q}=\min \left\{k-u_{1}, q, u_{n}-1\right\} \text {. }
$$

THEOREM 6.1. Let $f \in C^{1}[a, b]$ be given such that for the considered data set the system (3.1) is solvable. Then the error between $f$ and an interpolation spline $s$ of the form (2.4), (2.5) can be estimated by

$$
\left\|f^{(\nu)}-s^{(\nu)}\right\| \leqslant D_{\nu} \omega\left(f^{\prime}, h\right) \cdot h^{1-\nu} \quad \text { for } \nu=0,1
$$

where

$$
2 \cdot D_{0}=D_{1}=\frac{4 k-\tilde{q}}{\tilde{q}}+\frac{4 k(k-2)}{\tilde{q}(\tilde{q}+1)}
$$

Proof. With the defining equations of $\varphi_{j, i}$ we first obtain

$$
s^{\prime}(x)=m_{i-1}+a_{k, u_{i}} \cdot \int_{0}^{t} \xi^{k-u_{i}-1}(1-\xi)^{u_{i}-2}\left\{\lambda_{i} \xi+\rho_{i}\right\} d \xi, \quad x \in\left[x_{i-1}, x_{i}\right],
$$

where

$$
\begin{aligned}
\lambda_{i} & =\left(k-u_{i}\right) m_{i-1}+\left(u_{i}-1\right) m_{i}-(k-1) \tau_{i} \\
\rho_{i} & =\frac{k-u_{i}}{k}\left\{k \tau_{i}-\left(k-u_{i}+1\right) m_{i-1}-\left(u_{i}-1\right) m_{i}\right\}
\end{aligned}
$$

From the inequalities

$$
\frac{k \tau_{i}-\left(k-u_{i}\right) m_{i-1}}{u_{i}} \leqslant m_{i} \leqslant \frac{k \tau_{i}-\left(k-u_{i}+1\right) m_{i-1}}{u_{i}-1}
$$

it is easy to verify that

$$
\begin{gathered}
\frac{k-u_{i}}{u_{i}}\left(m_{i-1}-\tau_{i}\right) \leqslant \lambda_{i} \leqslant \tau_{i}-m_{i-1}, \\
0 \leqslant \rho_{i} \leqslant \frac{k-u_{i}}{u_{i}}\left(\tau_{i}-m_{i-1}\right) .
\end{gathered}
$$

Therefore, in view of $m_{i-1} \leqslant \tau_{i} \leqslant m_{i}$, we get after some calculations

$$
\left|s^{\prime}(x)-f^{\prime}(x)\right| \leqslant\left|m_{i-1}-f^{\prime}(x)\right|+\frac{k}{u_{i}-1} \cdot\left|\lambda_{i}\right|+\frac{k(k-1)}{\left(u_{i}-1\right)\left(k-u_{i}\right)} \rho_{i} .
$$


An analysis of $\left|\lambda_{i}\right|$ leads to

$$
\left|s^{\prime}(x)-f^{\prime}(x)\right| \leqslant\left|m_{i-1}-f^{\prime}(x)\right|+\frac{2 k\left(k-u_{i}\right)}{u_{i}\left(u_{i}-1\right)} \cdot\left(\tau_{i}-m_{i-1}\right)+\left(m_{i}-m_{i-1}\right) .
$$

Now, because of

$$
\frac{k \tau_{1}-u_{1} \tau_{2}}{k-u_{1}} \leqslant m_{0} \leqslant \tau_{1}, \tau_{n} \leqslant m_{n} \leqslant \frac{k \tau_{n}-\left(k-u_{n}+1\right) \tau_{n-1}}{u_{n}-1}
$$

and $m_{i-1} \leqslant \tau_{i} \leqslant m_{i}(i=1,2, \ldots, n)$, the following estimates are valid:

$$
\begin{gathered}
\left|m_{i-1}-f^{\prime}(x)\right| \leqslant\left\{\begin{array}{l}
\frac{u_{1}}{k-u_{1}}\left(\tau_{2}-\tau_{1}\right)+\left|\tau_{1}-f^{\prime}(x)\right| \leqslant \frac{k+u_{1}}{k-u_{1}} \cdot \omega\left(f^{\prime}, h\right) \quad \text { if } i=1, \\
\left|\tau_{i-1}-f^{\prime}(x)\right| \leqslant 2 \cdot \omega\left(f^{\prime}, h\right) \quad \text { if } i \in\{2,3, \ldots, n\},
\end{array}\right. \\
\tau_{i}-m_{i-1} \leqslant\left\{\begin{array}{l}
\frac{u_{1}}{k-u_{1}}\left(\tau_{2}-\tau_{1}\right) \leqslant 2 \cdot \frac{u_{1}}{k-u_{1}} \cdot \omega\left(f^{\prime}, h\right) \quad \text { if } i=1, \\
\left(\tau_{i}-\tau_{i-1}\right) \leqslant 2 \cdot \omega\left(f^{\prime}, h\right) \quad \text { if } i \in\{2,3, \ldots, n\},
\end{array}\right. \\
m_{i}-m_{i-1} \leqslant\left\{\begin{array}{l}
\tau_{2}-\frac{k \tau_{1}-u_{1} \tau_{2}}{k-u_{1}} \leqslant \frac{2 k}{k-u_{1}} \omega\left(f^{\prime}, h\right) \quad \text { if } i=1, \\
\tau_{i+1}-\tau_{i-1} \leqslant 3 \cdot \omega\left(f^{\prime}, h\right) \quad \text { if } i \in\{2,3, \ldots, n-1\}, \\
\frac{k \tau_{n}-\left(k-u_{n}+1\right) \tau_{n-1}-\tau_{n-1} \leqslant \frac{2 k}{u_{n}-1} \cdot \omega\left(f^{\prime}, h\right) \quad \text { if } i=n .1}{u_{n}-1} .
\end{array}\right.
\end{gathered}
$$

Finally, the bounds

$$
\begin{gathered}
\max \left\{\frac{k+u_{1}}{k-u_{1}}, 2\right\} \leqslant \frac{2 k-\tilde{q}}{\tilde{q}}, \quad \max \left\{\frac{2 k}{k-u_{1}}, 3, \frac{2 k}{u_{n}-1}\right\} \leqslant \frac{2 k}{\tilde{q}}, \\
\max \left\{\frac{4 k}{u_{1}-1}, \frac{4 k\left(k-u_{i}\right)}{u_{i}\left(u_{i}-1\right)},(i=2,3, \ldots, n)\right\} \leqslant \frac{4 k(k-2)}{\tilde{q}(\tilde{q}+1)}
\end{gathered}
$$

give (6.1) for $\nu=1$. The estimate (6.1) for $\nu=0$ can be obtained from this by standard techniques. Q.E.D.

THEOREM 6.2. Let $f \in C[a, b]$ be given such that for the considered data set the system (3.1) is solvable. With the abbreviation

$$
\Omega(\pi)=\max \left\{h_{i} / h_{j},|i-j|=1\right\}
$$

we obtain for a convex interpolation spline of the form (2.4), (2.5) the estimate

$$
\|f-s\| \leqslant C_{0} \cdot \omega(f, h)
$$

with $C_{0}=2\{k+(k-\tilde{q}) \Omega(\pi) / \tilde{q}\}+1$.

Proof. By virtue of (2.4), (2.5) we get

$$
|s(x)-f(x)| \leqslant\left|f_{i-1}-f(x)\right|+\left|m_{i-1}\right| h_{i}+\left|m_{i}\right| h_{i}, \quad x \in\left[x_{i-1}, x_{i}\right] .
$$


Further, from (6.6) there follows

$$
\begin{aligned}
& \left|m_{i-1}\right| h_{i} \leqslant\left\{\begin{array}{l}
\max \left\{\left|\tau_{1}\right|, \frac{\left|k \tau_{1}-u_{1} \tau_{2}\right|}{k-u_{1}}\right\} h_{1} \leqslant\left(k+\frac{u_{1}}{k-u_{1}} \cdot \frac{h_{1}}{h_{2}}\right) \cdot \omega(f, h) \quad \text { if } i=1, \\
\max \left\{\left|\tau_{i-1}\right|,\left|\tau_{i}\right|\right\} h_{i} \leqslant \max \left\{1, \frac{h_{i}}{h_{i-1}}\right\} \cdot \omega(f, h) \quad \text { if } i \in\{2, \ldots, n\},
\end{array}\right. \\
& \left|m_{i}\right| h_{i} \leqslant\left\{\begin{array}{l}
\max \left\{\left|\tau_{i}\right|,\left|\tau_{i+1}\right|\right\} h_{i} \leqslant \max \left\{1, \frac{h_{i}}{h_{i+1}}\right\} \cdot \omega(f, h) \quad \text { if } i \in\{1, \ldots, n-1\}, \\
\max \left\{\left|\tau_{n}\right|, \frac{\left|k \tau_{n}-\left(k-u_{n}+1\right) \tau_{n-1}\right|}{u_{n}-1}\right\} h_{n} \\
\leqslant\left(k+\frac{k-u_{n}+1}{u_{n}-1} \cdot \frac{h_{n}}{h_{n-1}}\right) \omega(f, h), \quad \text { if } i=n .
\end{array}\right.
\end{aligned}
$$

This leads to (6.7). Q.E.D.

In the case of monotone increasing and convex spline interpolation the dependence of $C_{0}$ on $\Omega(\pi)$ can be removed.

THEOREM 6.3. Let $f \in C[a, b]$ be given such that for the corresponding data set $a$ monotone increasing and convex interpolation spline of the form (2.4), (2.5) can be found. Then the error between $f$ and such a spline $s$ is bounded by

$$
\|f-s\| \leqslant C_{1} \cdot \omega(f, h)
$$

with $C_{1}=\max \{7 / 2,1+2(k-1) / \tilde{q}\}$.

Proof. Starting from the representation

$$
s(x)=f_{i-1}+m_{i-1} h_{i} t+a_{k, u_{i}} \cdot \int_{0}^{t} \int_{0}^{\eta} \xi^{k-u_{i}-1}(1-\xi)^{u_{i}-2}\left(\lambda_{i} \xi+\rho_{i}\right) d \xi d \eta
$$

for $x \in\left[x_{i-1}, x_{i}\right]$, with $\lambda_{i}, \rho_{i}$ from (6.2), (6.3), and using the identity

$$
\int_{0}^{1} \int_{0}^{\eta} \xi^{\nu}(1-\xi)^{\mu} d \xi d \eta=\frac{\mu+1}{\mu+\nu+2} \int_{0}^{1} \xi^{\nu}(1-\xi)^{\mu} d \xi
$$

for arbitrary integers $\mu, \nu$ (see [15]), we obtain after straightforward calculation

$$
|s(x)-f(x)| \leqslant\left|f_{i-1}-f(x)\right|+m_{i-1} h_{i}+\left|\lambda_{i}\right| h_{i}+\frac{k}{k-u_{i}} \cdot \rho_{i} h_{i} .
$$

Finally, (6.4), (6.5) and $m_{i-1} \geqslant 0$ yield

$$
\begin{aligned}
|s(x)-f(x)| & \leqslant \omega(f, h)+\left|\lambda_{i}\right| h_{i}+\frac{k}{u_{i}} \cdot \omega(f, h) \\
& \leqslant\left[1+\frac{k}{u_{i}}+\max \left\{\frac{k-u_{i}}{u_{i}}, 1\right\}\right] \cdot \omega(f, h) \leqslant C_{1} \cdot \omega(f, h) \text {. Q.E.D. }
\end{aligned}
$$

Remark. With Theorem 6.1 we have obtained a better error estimate than that given by Neuman in [12], [13] for special cases.

Sektion Mathematik

Technische Universität Dresden

Mommsenstrasse 13

DDR-8027 Dresden, German Democratic Republic 
1. C. DE Boor \& B. SWARTZ, "Piecewise monotone interpolation," J. Approx. Theory, v. 21, 1977, pp. $411-416$.

2. W. Burmeister, W. Hess \& J. W. SChmidt, "Convex spline interpolants with minimal curvature," Computing, v. 35, 1985, pp. 219-229.

3. L. E. Deimel, D. F. McAllister \& J. A. Roulier, Smooth Curve Fitting With Shape Preservation Using Osculatory Quadratic Splines, Proc. 11th Annual Conf. on the Interface Between Statistics and Computer Sci. (A. R. Gallant and T. M. Gerig, eds.), Institute of Statistics, North Carolina State University, 1978, pp. 343-347.

4. B. Dimsdale, “Convex cubic splines,” IBM J. Res. Develop., v. 22, 1978, pp. 168-178.

5. D. F. McAllister, E. Passow \& J. A. Roulier, “Algorithms for computing shape preserving spline interpolations to data," Math. Comp., v. 31, 1977, pp. 717-725.

6. H. MetTKe, “Convex cubic Hermite-spline interpolation," J. Comput. Appl. Math., v. 9, 1983, pp. 205-211 and v. 11, 1984, pp. 377-378.

7. H. Metrke, "Quadratische Splineinterpolation bei zusammenfallendem Interpolations- und Splinegitter,” Beiträge Numer. Math., v. 8, 1980, pp. 113-119.

8. H. MetTke \& T. Lingner, “Ein Verfahren zur konvexen kubischen Splineinterpolation,” Wiss. Z. Tech. Univ. Dresden, v. 32, 1983, pp. 77-80.

9. E. Neuman, "Convex interpolating splines of arbitrary degree," in Numerical Methods of Approximation Theory, Vol. 5 (L. Collatz, G. Meinardus and H. Werner, eds.), Birkhäuser Verlag, Basel, 1980, pp. $211-222$.

10. E. NeUman, “Convex interpolating splines of arbitrary degree. II,” BIT, v. 22, 1982, pp. 331-338.

11. E. Neuman, "Convex interpolation splines of odd degree," Utilitas Math., v. 14, 1978, pp. 129-140.

12. E. Neuman, Shape Preserving Interpolation by Polynomial Splines, Report no. N-112, Institute of Computer Science, University of Wroclaw, 1982.

13. E. Neuman, "Uniform approximation by some Hermite interpolating splines," J. Comput. Appl. Math., v. 4, 1978, pp. 7-9.

14. E. PAssow, "Monotone quadratic spline interpolation," J. Approx. Theory, v. 19, 1977, pp. $143-147$.

15. E. Passow \& J. A. Roulier, "Monotone and convex spline interpolation," SIAM J. Numer. Anal., v. 14, 1977, pp. 904-909.

16. J. A. Roulier, “Constrained interpolation," SIAM J. Sci. Statist. Comput., v. 1, 1980, pp. $333-344$.

17. J. W. Schmidt \& W. Hess, "Schwach verkoppelte Ungleichungssysteme und konvexe Splineinterpolation," Elem. Math., v. 39, 1984, pp. 85-95.

18. B. K. Swartz \& R. S. VARga, "Error bounds for spline and L-spline interpolation," J. Approx. Theory, v. 6, 1972, pp. 6-49. 